\title{
The magnetic properties of single-crystalline atacamite, $\mathrm{Cu}_{2} \mathrm{Cl}(\mathrm{OH})_{3}$
}

\author{
L. Heinze ${ }^{\mathrm{a}, 1}$, , R. Beltran-Rodriguez ${ }^{\mathrm{b}}$, G. Bastien ${ }^{\mathrm{b}}$, A.U.B. Wolter ${ }^{\mathrm{b}}$, M. Reehuis $^{\mathrm{c}}$, J.-U. Hoffmann ${ }^{\mathrm{c}}$, K.C. Rule ${ }^{\mathrm{d}}$, \\ S. Süllow ${ }^{\mathrm{a}}$ \\ ${ }^{a}$ Institut für Physik der Kondensierten Materie, TU Braunschweig, 38106 Braunschweig, Germany
${ }^{b}$ Leibniz-Institut für Festkörper- und Werkstofforschung IFW Dresden, 01069 Dresden, Germany
${ }^{c}$ Helmholtz-Zentrum Berlin für Materialien und Energie, 14109 Berlin, Germany \\ ${ }^{d}$ Australian Centre for Neutron Scattering, ANSTO, Kirrawee DC, New South Wales 2234, Australia
}

\begin{abstract}
We present susceptibility measurements on the natural mineral atacamite, $\mathrm{Cu}_{2} \mathrm{Cl}(\mathrm{OH})_{3}$, for the first time along the three crystallographic axes. Further, we have carried out an elastic neutron diffraction experiment which shows that the symmetry of the magnetic ground state of atacamite is described by a propagation vector $\mathbf{q}=(1 / 201 / 2)$.
\end{abstract}

Keywords:

quantum magnetism, frustration, low-dimensional magnetism, elastic neutron diffraction

The natural mineral atacamite, $\mathrm{Cu}_{2} \mathrm{Cl}(\mathrm{OH})_{3}$, in its orthorhombic form (space group: Pnma, lattice parameters: $a=6.030 \AA, b=6.865 \AA, c=9.120 \AA)[1,2]$, has been reported to exhibit magnetic behavior characteristic of a frustrated quantum magnet $[3,4]$. Notably, an antiferromagnetic transition at $T_{N}=9.0 \mathrm{~K}$ has been observed, further, previous susceptibility measurements indicate a Curie-Weiss temperature $\left|\Theta_{C W}\right| \gg T_{N}$. So far, attempts have been undertaken to determine the symmetry of the magnetic ground state of this material by means of $\mu \mathrm{SR}$ and NMR measurements $[4,5]$. The conclusions drawn from these studies, however, are contradictory: From the $\mu \mathrm{SR}$ measurements on mineral atacamite, a disordered state was suggested [4], whereas from the proton NMR spectra of synthetic samples an all-in all-out alignment of the $\mathrm{Cu}$ magnetic moments on crystallographic inequivalent sites was deduced $\left(\mu_{I}\right.$ $=1.12 \mu_{B}$ and $\mu_{I I}=0.25 \mu_{B}$ ), similar to the magnetic structure of an antiferromagnetic pyrochlore lattice [5]. Starting from this given situation, we have reinvestigated the magnetic properties of atacamite. Two singlecrystalline, mineral samples with the dimensions of about $2 \mathrm{~mm} \times 5 \mathrm{~mm} \times 2 \mathrm{~mm}$ (origin: Poona Mine, Moonta, Australia) from the same crystal batch were studied by means of susceptibility measurements performed with the magnetic field applied along each of the three crystallographic axes and elastic neutron diffraction.

Email address: 1.heinze@tu-braunschweig.de (L. Heinze)
The susceptibility measurements were carried out with a magnetic field of $0.1 \mathrm{~T}$ (field cooled (fc)) applied along the three crystallographic axes within the temperature range of 1.8 - $300 \mathrm{~K}$ using a commercial SQUID magnetometer. Fig. 1 shows the results of the temperature dependent susceptibility measurements (a) together with the inverse susceptibility $\chi^{-1}$ as a function of temperature (b) for sample $1(42 \mathrm{mg})$. In accordance with Refs. [3, 4], an antiferromagnetic transition can be observed around $9.0 \mathrm{~K}$. Additionally, some anisotropy of the temperature dependence of the susceptibility can be observed. The solid lines in Fig. 1 (b) are linear fits of the high temperature data of $\chi^{-1}(T)$ in order to extract the Curie-Weiss behavior following $\chi^{-1} \propto\left(T-\Theta_{C W}\right)$, with the Curie-Weiss temperature $\Theta_{C W}$. While there is a slight uncertainty in the determination of $\Theta_{C W}$ because of the limited fit range $200 \mathrm{~K} \leq T \leq 300 \mathrm{~K}$, this way, we have obtained values $\Theta_{C W}$ of $-136 \mathrm{~K}$ along the $a$ axis, $-103 \mathrm{~K}$ along $b$ and $-112 \mathrm{~K}$ along the $c$ axis (see Fig. 1 (b)). The behavior is fully consistent with the susceptibility measured for polycrystalline material in Ref. [4]. Considering the transition temperature $T_{N}=9.0 \mathrm{~K}$, these experimental results lead to a ratio $\left|\Theta_{C W} / T_{N}\right|>$ 10. This value is often used as indicator for magnetic frustration and/or low magnetic dimensionality within a system and shows that these phenomena have to be taken into account for atacamite.

Elastic neutron diffraction measurements were carried out using the flat-cone diffractometer E2 of the 

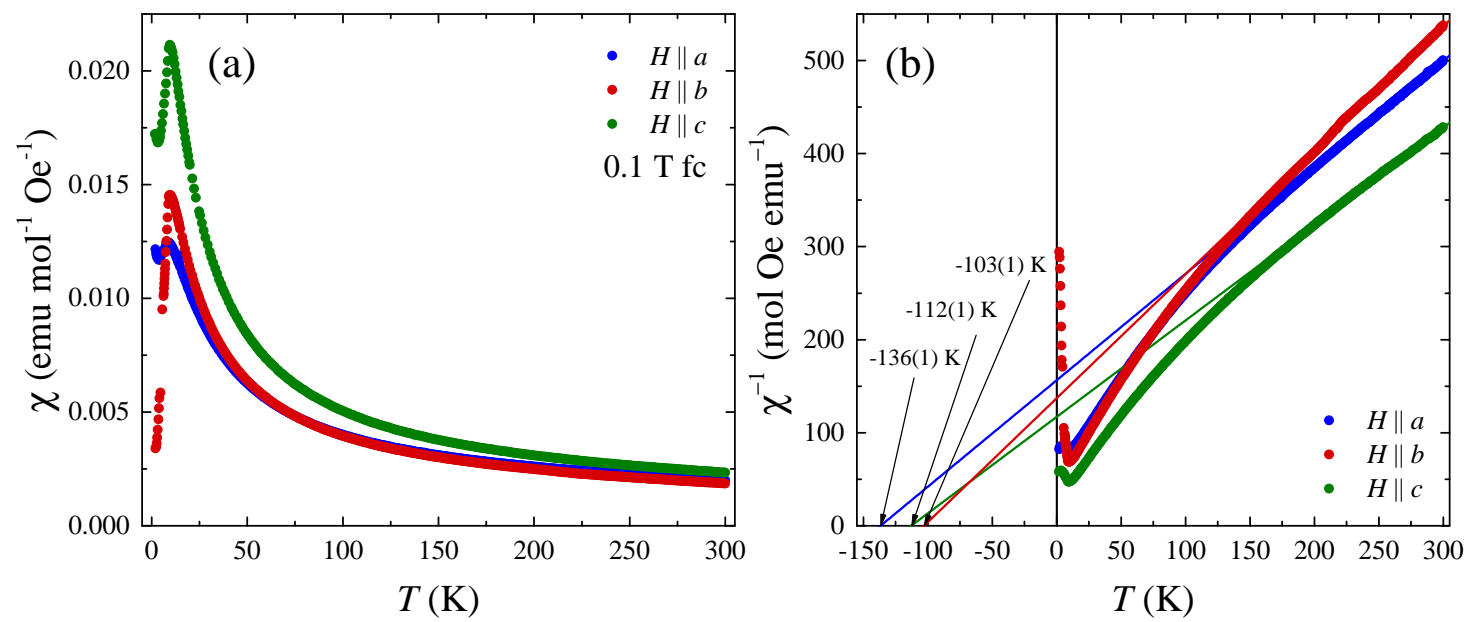

Figure 1: Magnetic susceptibility along the three crystallographic axes of a mineral atacamite sample for a magnetic field of $0.1 \mathrm{~T}$ (a) together with the inverse susceptibility as function of temperature (b). The solid lines added to the plot of $\chi^{-1}(T)$ in (b) are linear fits of the high temperature data $(T>200 \mathrm{~K})$ according to a Curie-Weiss law; for details see text

Helmholtz-Zentrum Berlin für Materialien und Energie (HZB), Germany. The single-crystalline atacamite sample $2(39 \mathrm{mg})$ was oriented along the crystallographic $b$ axis and rotated within the $a c$ plane for the neutron diffraction experiment (neutron wavelength $\lambda=2.38 \AA$ ). This way, the $(h 0 l)$ scattering plane was scanned, once at $40 \mathrm{~K}$, i.e., far above the transition temperature $T_{N} \mathrm{ob}$ served for atacamite, and at base temperature $(1.6 \mathrm{~K})$ of the ${ }^{4} \mathrm{He}$ cryostat (Fig. 2). In addition to the nuclear Bragg peaks, when cooling below $T_{N}$, magnetic Bragg peaks are observed at $(1 / 201 / 2),\left(\begin{array}{lll}1 / 2 & 0 & 1 / 2\end{array}\right),(1 / 20$ $3 / 2),(1 / 205 / 2),(1 / 207 / 2)$ and a few weaker magnetic reflections at $(3 / 201 / 2)$ and $(3 / 20 \overline{1 / 2})$ (indicated by the red circles in Fig. 2). As from Fig. 2 these magnetic reflections are difficult to see, in the inset we include a rocking scan through the $(1 / 201 / 2)$ peak, clearly illustrating the presence of magnetic intensity. This set of magnetic reflections corresponds to a propagation vector $\mathbf{q}=(1 / 201 / 2)$, which means that the magnetic unit cell is double of the nuclear unit cell along the crystallographic $a$ and $c$ axis. This observation is in contradiction to the suggestion of a disordered ground state by Zheng et al. [4].

In conclusion, for the first time we have measured the magnetic susceptibility along the three crystallographic axes. From our data, in agreement with Refs. [3, 4], we find an antiferromagnetic transition around $T_{N}=9 \mathrm{~K}$. Further, we confirm that magnetic frustration plays an important role regarding the magnetic behavior of atacamite, however, we would like to emphasize that also low dimensionality can be important here. Addition- ally, by carrying out an elastic neutron diffraction experiment, we show that the symmetry of the magnetic ground state of atacamite is described by a propagation vector $\mathbf{q}=\left(\begin{array}{lll}1 / 2 & 0 & 1 / 2\end{array}\right)$. This result clearly contradicts the possibility of a disordered, glassy ground state for mineral atacamite from Ref. [4]. From our experimental data we cannot unambiguously decide whether the magnetic structure proposed in Ref. [5] is consistent with our magnetic propagation vector $\mathbf{q}$. In order to resolve this question, further single-crystal neutron diffraction experiments will be carried out in the near future, this way to firmly establish the ordered magnetic structure of atacamite.

\section{Acknowledgments}

We thank the HZB for the allocation of neutron radiation beamtime and thankfully acknowledge the financial support from HZB. Our work has been supported by the DFG under Grants No. WO 1532/3-2 and No. SU 229/9-2 and SFB 1143. We thank G. Paskalis for supplying us with both atacamite crystals and thank $\mathrm{Ch}$. Blum, K. Ivshin and O. Kataeva for orienting the crystals.

[1] J. B. Parise, B. G. Hyde, Acta Cryst., Sect. C: Cryst. Struct. Commun. 42, 1277 (1986)

[2] A. F. Wells, Acta Cryst. 2, 175 (1949).

[3] X. G. Zheng, E. S. Otabe, Solid State Commun. 130, 107 (2004).

[4] X. G. Zheng, T. Mori, K. Nishiyama, W. Higemoto, H. Yamada, K. Nishikubo, and C. N. Xu, Phys. Rev. B 71, 174404 (2005).

[5] K. Zenmyo, H. Kubo, M. Tokita, T. Hamasaki, M. Hagihala, X. G. Zheng, T. Kawae, Y. Takeuchi, and M. Matsumura, J. Phys. Soc. Jpn. 82, 084707 (2013). 

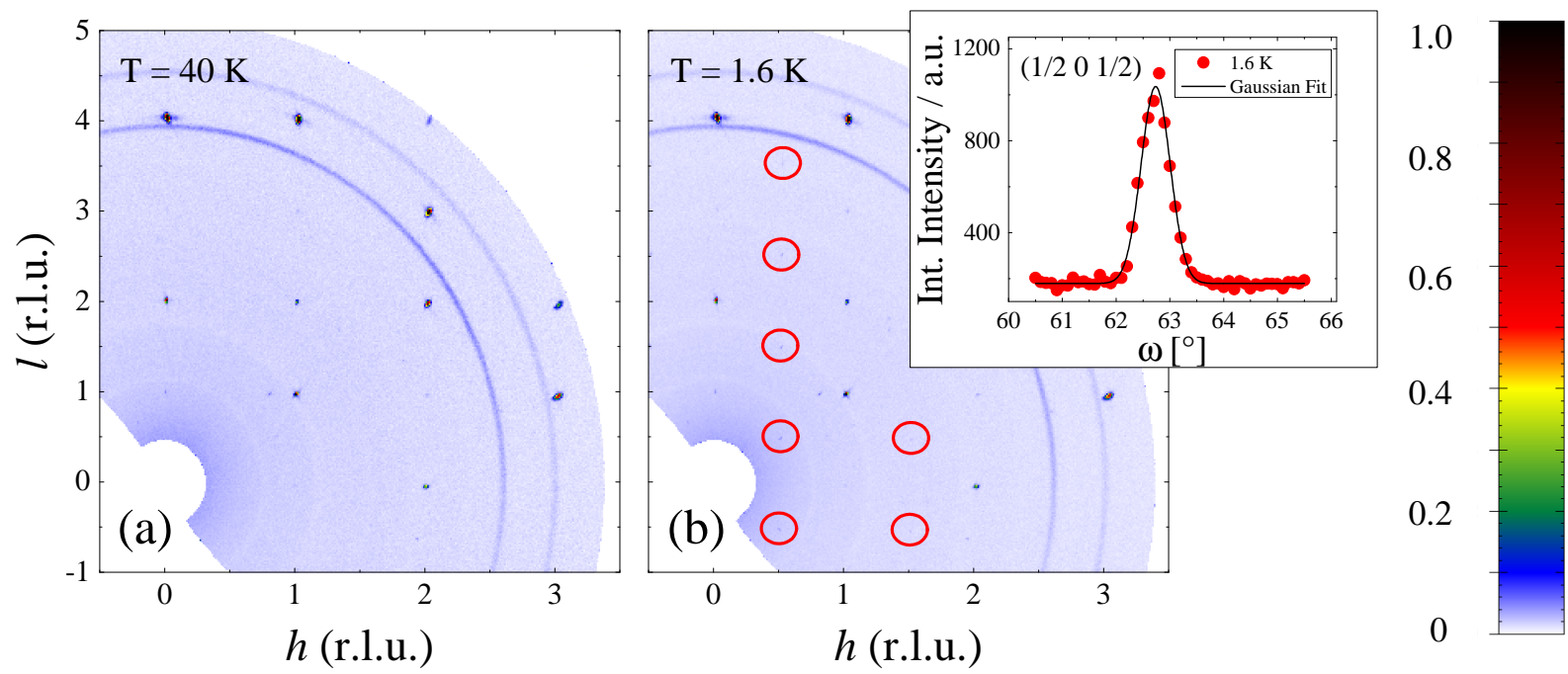

Figure 2: Elastic neutron diffraction pattern of single-crystalline atacamite in reciprocal space within the $(h 0 l)$ scattering plane. The scan at $40 \mathrm{~K}$ shows the nuclear Bragg peaks for the crystal symmetry of atacamite (a) whereas when cooling below $T_{N}$ magnetic reflections at (1/2 $\left.01 / 2\right),(1 / 20$ $\overline{1 / 2}),(1 / 203 / 2),(1 / 205 / 2)$ as well as weaker reflections at $(3 / 201 / 2)$ and $(3 / 20 \overline{1 / 2})$ can be observed within the scattering plane (b), as indicated by the red circles. The concentric circles of higher intensity appearing in both scans are due to the diffraction from the cryostat and the Al-sample holder. The inset in (b) displays an $\omega$-scan of the $(1 / 201 / 2)$ reflection with a step width of $\Delta \omega=0.1^{\circ}$ fitted using a Gaussian function. 